\title{
Tendencias de tarificación en el suministro de energía eléctrica
}

\author{
Pricing Trends in Electric Energy Supply
}

\author{
Hernando Durán Castro ${ }^{(1)}$ \\ (1) Ph. D. en Engineering Economic Systems, Universidad de Stanford. Profesor Titular, Universidad de los Andes. Bogotá, Colombia. \\ hduran@uniandes.edu.co
}

Recibido 4 de noviembre de 2014. Modificado 15 de enero de 2015. Aprobado 12 de febrero de 2015.

DOI: http://dx.doi.org/10.16924/riua.v0i41.686

\section{Palabras clave}

Costeo dinámico, costo marginal, energía eléctrica, medidores inteligentes, tarifas por tiempo de uso, promedio ponderado de costo de capital WACC

\section{Resumen}

Se presenta un modelo de precios de energía eléctrica que permite a productores y consumidores operar como mercado. La demanda de energía eléctrica es variable en el tiempo y no es almacenable de un período a otro: se genera en el momento en que se demanda. Las tecnologías de generación (hidráulica, nuclear, térmica, etc.) utilizan distintos combustibles y tienen costos diferentes de inversión en capacidad y de operación. El modelo minimiza la suma de ambos costos y garantiza que los productores obtengan un beneficio sobre la inversión en capacidad igual al WACC (Weighted Average Cost of Capital).

\section{Key words}

Dynamic pricing, electric power, marginal cost, smart meters, time of use pricing, weighted average cost of capital WACC.

\begin{abstract}
An electric power price model that permits producers and consumers to operate as a market is presented. Electric power is highly variable in time and cannot be stored from one period to another: it is generated when demanded. Generation technologies (hydraulic, nuclear, thermal, etc.) use different energy sources and have different capacity investment and operating costs. The model minimizes the sum of both costs insuring that producers obtain returns on capacity investment equal to WACC (Weighted Average Cost of Capital).
\end{abstract}

\section{INTRODUCCIÓN}

El desarrollo tecnológico permite hoy instalar contadores inteligentes de energía eléctrica (Smart meters) que miden y acumulan el consumo horario, se comunican doble vía con la empresa suministradora, y reciben de ella con anticipación horaria oferta de precios de la energía, lo cual permite a los usuarios tomar decisiones en tiempo real sobre su consumo (Borenstein, 2005; Joskow, 2012).

Estos avances en medición del consumo, intercambio de información entre consumidores y productores, y facturación de energía al usuario, en tiempo real, hacen posible que el suministro de potencia eléctrica pueda funcionar como mercado. Los productores fijan precios periódicamente, por ejemplo, cada hora en cada nodo del sistema eléctrico, y los consumidores deciden cuánta potencia consumen en esa hora dependiendo del precio en el sitio de consumo.
Por otro lado, la congestión en el sistema de suministro de energía eléctrica en las horas de mayor consumo hace patente la conveniencia de asignar precios a los usuarios que reconozcan la hora en que ocurre la demanda, como origen de los costos marginales de expansión en capacidad en que incurren los productores y que incentive a los primeros a desplazar su demanda hacia momentos de menor congestión (Bye, 1926; Boiteaux, 1949; Crew, 1995).

El mercado de potencia eléctrica logra acercarse hoy a metas de eficiencia económica inalcanzables antes. Idealmente, los precios de la potencia deben reflejar los costos marginales de generación y transmisión en cada hora y nodo del sistema (Steiner, 1957). Es necesario que con estos precios los productores recuperen no solamente los costos de producción sino los costos de inversión en capacidad de producción más la utilidad. 
Millones de hogares y oficinas en ciudades de Canadá, Estados Unidos, Europa y China cuentan hoy con este tipo de facturación, para mencionar tan solo algunos de los países que han liderado la implementación de lo que se conoce como "tiempo de uso" (Time Of Use), y "Costeo Dinámico" (Dynamic Pricing) de la energía eléctrica (Joskow, 2012).

El presente artículo describe un modelo de tarificación del suministro de energía eléctrica estudiado por el autor (Durán, 2001); tiene en cuenta los costos marginales de inversión y de producción, el intercambio de información en tiempo real y el uso de contadores inteligentes de energía eléctrica (Smart Meters). Se muestra que con precios iguales a costos marginales de producción más un cargo adicional igual al costo marginal de inversión en capacidad con la tecnología que funciona solamente durante el período de mayor consumo, los productores con todas las tecnologías recuperan sus costos de inversión y operación.

\section{BASES ECONÓMICAS Y REGULATORIAS}

Los productores, como inversionistas, persiguen lucro que tratándose de un bien público está sujeto a regulación. El regulador limita este lucro a aquel que resulta de aplicar la tasa de interés WACC (Weighted Average Cost of Capital) sobre los costos de inversión en capacidad de producción del bien. Las tarifas que se cobran al consumidor final no deben entonces superar a las necesarias para recuperar los costos de producción (combustibles, salarios de operadores de la planta, etc.) más los costos de inversión, incluido dentro de estos la utilidad WACC.

\section{COSTOS MARGINALES DE INVERSIÓN Y OPERA- CIÓN}

El sector de oferta se supone que está compuesto por diferentes tecnologías de generación de potencia eléctrica con costos unitarios fijos de inversión y de operación. Cada tecnología se caracteriza por su costo de capacidad $(\$ / K W)$ y costo de generación $(\$ / K W H)$. La energía eléctrica (como corriente alterna) no se puede almacenar de un período de tiempo a otro; se genera y transmite en cada instante en la cantidad de potencia eléctrica que se demanda. La Figura 1 ilustra costos típicos de tecnologías hidráulica, de carbón, y de gas de ciclo abierto y de ciclo combinado ${ }^{1}$.

\section{Demanda}

El modelo de precios de tiempo de uso considera que la demanda de potencia eléctrica es variable en el tiempo. La demanda agregada de potencia presenta altibajos y picos durante el ciclo considerado $T$; la función de demanda de potencia eléctrica $D(t)$ se define como el nivel de potencia en $t$.

\section{OFERTA}

Existen $m$ tecnologías de producción de energía eléctrica $1 \leq$ $j \leq m$ con costos unitarios fijos de capacidad y de producción dados por:

$I_{j}=$ Costo (anualizado) de inversión en capacidad de producción correspondiente al período $T$ con la tecnología $j$. El valor óptimo de la capacidad de la tecnología $j$ se denomina Yj.

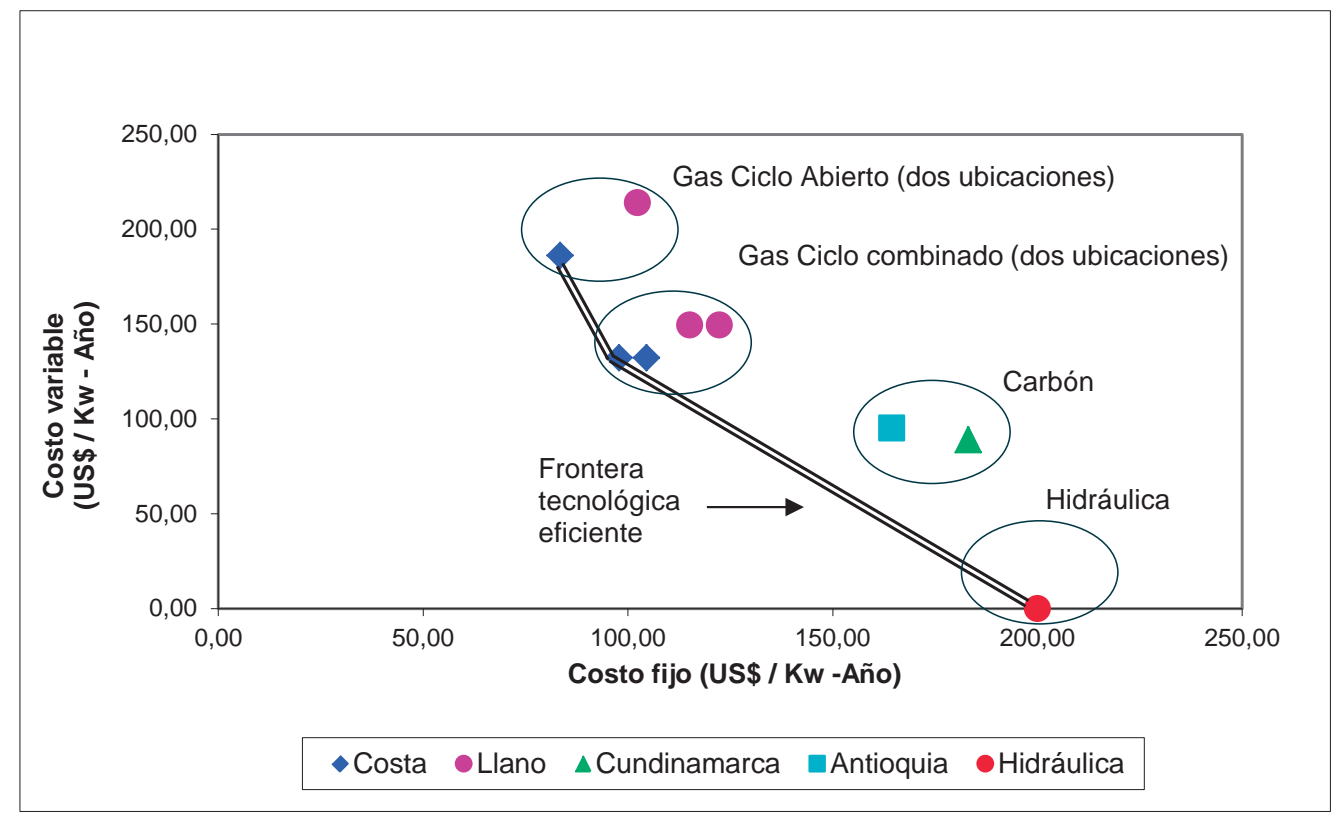

Figura 1. Costos de generación.

1 Datos de tecnologías de generación eléctrica en Colombia, año 2000 
$C_{j} Z_{j}(t)=$ Costo de producción de la potencia eléctrica en el instante $t Z_{j}(t)$ con la tecnología $j$. Los costos unitarios de capacidad y de producción $I_{j}$ y $C_{j}$, respectivamente, son constantes y diferentes para cada tecnología. Es importante resaltar que los costos al consumidor dependen también de la ubicación de la demanda con relación a las respectivas plantas de generación, haciendo necesario añadir en cada caso los costos de transformación a alta tensión y de transmisión correspondientes.

\section{Frontera Tecnológica Eficiente}

Las tecnologías se numeran por orden creciente de costo unitario de generación:

$$
C_{1}<C_{2}<\ldots<C_{\mathrm{m}}
$$

Satisfacen, además, las siguientes condiciones de eficiencia económica: a) tecnologías con ambos costos, de inversión y de producción, mayores que los correspondientes de otra, están dominadas económicamente por ésta y se descartan:

$$
I_{1}>I_{1}>\ldots>I_{m}
$$

b) pendientes decrecientes de la frontera al aumentar el costo de producción (convexidad de la frontera), es decir:

$$
T>T_{1}>T_{2}>\ldots>T_{m-1}>0
$$

donde

$$
T_{j}=\left(I_{j}-I_{j+1}\right) /\left(C_{j+1}+C_{j}\right)
$$

para $j=1,2, \ldots . . m-1 ; T_{j}$ indica el tiempo de utilización óptima a plena capacidad de la tecnología con subíndice $j$. La frontera tecnológica eficiente de las tecnologías en el caso de la Figura 1 se muestra con línea doble y contiene solamente tres de ellas: Gas Ciclo Abierto (A), Gas Ciclo Combinado (C) e Hidráulica $(\mathrm{H})$ por orden decreciente de costo unitario de producción.

Las tecnologías a Carbón están dominadas por la frontera tecnológica (combinaciones lineales de Gas Ciclo Combinado e Hidráulica. Sin embargo, podrían llegar a ser eficientes dependiendo de la ubicación de la demanda si se tienen en cuenta los costos de transmisión (Demandas vecinas a las minas de carbón).

\section{FORMULACIÓN DEL PROBLEMA}

El problema es Objetivo: Minimizar

$$
\sum_{j=1}^{M} I_{j} Y_{j}+\sum_{j=1}^{M} C_{j} \int_{t=0}^{T} Z_{j}(t) d t
$$

Restricciones:

Suministro de la demanda en $t$ :

$$
D(T)-\sum_{j=1}^{M} Z_{j}(t)=0
$$

No-negatividad de la producción en $t$ :

$$
Z_{j}(t) \geq 0 \quad j=1,2 \ldots M
$$

Restricciones de capacidad en $t$ :

$$
Z_{j}(t) \leq Y_{j} \quad j=1,2 \ldots M
$$

\section{Resultados}

\section{COMBINACIÓN ÓPTIMA DE TECNOLOGÍAS}

La combinación óptima de tecnologías satisface la condición de que la suma de las capacidades óptimas de las tecnologías hasta la $j, Y_{1}+Y_{2}+\cdots Y_{j}$ sea igual al nivel de potencia que es excedido por la demanda durante intervalos de tiempo en el período $T$ de duración total igual a $T_{j}$.

\section{DESPACHO ÓPTIMO POR ORDEN DE MÉRITO}

Si $Z_{j}(t)>0$ en el tiempo $t$, entonces $Z_{k}(t)=Y_{k}$ para todo $k<$ $j$. Las tecnologías se despachan por orden creciente de costo unitario de producción hasta copar su capacidad, y así hasta suplir la demanda. En cada tiempo $t$ hay una sola tecnología $j$ tal que $0<Z_{j}(t)<Y_{j}$. Esta tecnología se denomina la tecnología en el margen en el tiempo $t$.

\section{Precios óptimos}

La estructura óptima de precios consta de un cargo por Potencia consumida en el tiempo $t$ más un cargo por capacidad por unidad de potencia consumida en el momento en que ocurre la demanda máxima (pico) en el ciclo. 
El precio óptimo de la potencia en $t$ es igual al costo unitario de producción (costo marginal) con la tecnología $j$ que está en el margen en ese momento:

$$
P(t)=C_{j}
$$

El cargo óptimo por capacidad es igual al costo unitario de capacidad de la tecnología utilizada para pasar el pico de la demanda.

$$
\hat{P}=I_{m}
$$

\section{RECUPERACIÓN DE COSTOS DE CADA TECNOLOGÍA}

Los precios óptimos de potencia satisfacen la siguiente relación, para $j<m$ :

$$
\int_{0}^{T_{j}}\left(P(t)-C_{j}\right) d t+I_{m}=I_{j}
$$

De (12) se desprende que los costos de capacidad y de producción con la tecnología $j$ se recuperan exactamente con los ingresos percibidos al cobrar los precios de potencia $P(t)$ a la demanda $Z_{j(t)} \Delta t$ en cada intervalo del periodo $T \mathrm{y}$ el precio de capacidad $\hat{P}=I_{m}$ a la potencia producida en el momento del pico de la demanda.

Esta propiedad de los precios óptimos reconcilia dos de los objetivos fundamentales de la teoría de precios de un servicio público, a saber: eficiencia económica en razón a que los precios óptimos son los costos marginales de producción y recuperación de los costos totales de los productores.

Ejemplo. Tres tecnologías (costos fijos y variables de Tabla siguiente)

Tabla 1. Costos fijos y variables de las tres tecnologías

\begin{tabular}{|c|c|c|}
\hline Tecnología & $\begin{array}{c}\text { Costo Fijo } \\
\text { (US\$̦/KW-AÑo) }\end{array}$ & $\begin{array}{c}\text { Costo Variable } \\
\text { (US\$̦/KW-AÑo) }\end{array}$ \\
\hline H & 200 & 0 \\
\hline GCC & 120 & 100 \\
\hline GCA & 60 & 200 \\
\hline
\end{tabular}

$T_{H}=\frac{I_{H}-I_{C}}{C_{C}-C_{H}}=\frac{200-120}{100-0}=0,8 ; \quad T_{C}=\frac{I_{C}-I_{A}}{C_{A}-C_{C}}=\frac{120-60}{200-100}=0,6 ;$
Recaudo de la tecnología C:

$\left(C_{A}-C_{C}\right) T_{C}+I_{A}=(200-100) 0.6+60=120=I_{C}$

Recaudo de la tecnología $\mathrm{H}$

$\left(C_{A}-C_{H}\right) T_{C}+\left(C_{C}-C_{H}\right)\left(T_{H}-T_{C}\right)+I_{A}=200 * 0.6+100 * 0.2+60=200=I_{H}$

\section{MERCAdo MAYORISTA DE ENERGíA ELÉCTRICA}

Cuando la capacidad máxima disponible en el mercado de una tecnología $K_{j}$ es diferente a la capacidad óptima $Y_{j}$ determinada arriba, se puede presentar alguno de los siguientes casos: si $K_{j}<Y_{j}$ la tecnología $j$ está sub-instalada con respecto al óptimo; si $K_{j}>Y_{j}$, la tecnología esta sobre-instalada. En el primer caso, el tiempo de operación a plena capacidad es $T_{j}^{\prime}>T_{j}$. En el período $\left(T_{j}, T_{j}^{\prime}\right)$ la tecnología opera a plena capacidad en lugar de operar en el margen. Los consumidores pagan entonces un costo adicional igual a $\left(T_{j}^{\prime} T_{j}\right) K_{j}\left(C_{j+1}-C_{j}\right)$ con respecto al óptimo, que se traslada como excedente a los productores con la tecnología $j$. Se incentivan en consecuencia los inversionistas de tecnología $j$ para incrementar su capacidad capturando parte o el total del excedente. En la medida en que se cubra la sub-instalación la franja $\left(T_{j}, T^{\prime}\right)$ se reduce y el excedente retorna a su valor de cero sin sub-instalación. Esta situación se puede calificar como "perversa” aduciendo que hay que esperar a que haya sub-instalación de cualquier tecnología para que los inversionistas en el mercado tengan incentivo para incrementar su capacidad pues, así cuentan con una renta cautiva. Esto sería económicamente correcto si esas rentas fuesen su única fuente de utilidad, es decir, si no existiese una adecuada tasa de retorno sobre la inversión incluida dentro del costo unitario de capacidad $I_{j}$, como es el WACC.

En el caso en que $K_{j}>Y_{j}$ la tecnología $j$ se despacha a plena capacidad durante un tiempo menor que el óptimo. El inversionista pierde el ingreso correspondiente al tiempo en que la tecnología se despacha en el margen cuando en el óptimo se despacharía a plena capacidad. Con una tasa adecuada de retorno los inversionistas tienen incentivo económico para instalar justamente la capacidad óptima de cada tecnología.

\section{TARIFAS ÓPTIMAS AL CONSUMIDOR}

Muchas empresas de suministro de energía eléctrica cuentan hoy con sistemas de medición y facturación en tiempo real (Smart metering and accounting), en los cuales el contador de energía de cada consumidor es inteligente, está conectado a la red de internet, y efectúa automáticamente la facturación. A su vez, el consumidor cuenta en tiempo real con toda la información, los datos y las estadísticas de variación en el tiempo de su consumo y de los precios de la energía, y toma en tiempo real las decisiones de consumo. Existe una clara 
tendencia a migrar hacia estas nuevas tecnologías. Sin embargo, sus costos de implementación versus continuar utilizando la infraestructura existente harán que dicha migración no ocurra de inmediato, particularmente en países en vía de desarrollo.

\section{Conclusiones}

Se muestra la interacción entre los costos de inversión en capacidad y los de producción, y entre los problemas de inversión a largo plazo, de operación a corto plazo y los precios de energía y de potencia en los diferentes instantes del ciclo.

El precio de la energía eléctrica en cada momento es igual al costo marginal de producción con la tecnología que se despacha en el margen.

El costo marginal durante el pico de la demanda incluye adicionalmente el costo marginal de añadir capacidad al sistema con la tecnología que se despacha en el pico.

El cobro óptimo del servicio requiere de un sistema de medición del consumo de energía en tiempo real que calcule el precio óptimo correspondiente y ejecute la facturación (Smart metering and accounting).

Cada tecnología recupera su costo de inversión en capacidad, incluida la tasa de retorno (WACC), con el excedente entre el precio de la potencia y el costo de producción durante el tiempo en que la tecnología funciona a plena capacidad.

\section{Apendice - Derivación de los Resultados}

\section{CONDiciones DE OPTIMALIDAd (VER LUENBERGeR POR EJEMPLO)}

Introduciendo multiplicadores $-\mu_{\mathrm{j}}^{-}(t), \mu_{\mathrm{j}}^{+}(t)$ de las restricciones 7 y 8 y considerando que $P(t)$, el precio de la unidad de potencia, es igual al costo marginal de satisfacer una unidad adicional de la demanda $D(t)$, se tiene:

El Lagrangiano es: con las siguientes condiciones de holgura complementaria:

$$
\begin{aligned}
& Z_{j}(t)>0 \Rightarrow \mu_{j}^{-}(t)=0 ; \\
& \mu_{j}^{-}(t)>0 \Rightarrow Z_{j}(t)=0 ; \\
& Z_{j}(t)<Y_{j} \Rightarrow \mu_{j}^{+}(t)=0 ; \\
& \mu_{j}^{+}(t)>0 \Rightarrow Z_{j}(t)=Y_{j} ;
\end{aligned}
$$

Sean $T^{-}, T, T^{+}$los intervalos de tiempo en que la tecnología $j$ opera a nivel cero, en el margen, y a plena capacidad respectivamente:

$Z_{j}(t)=0 ; 0<Z_{j}(t)<Y_{j} ; Z_{j}(t)=Y_{j} ;$

de A3 se desprende:

$$
\mu_{j}^{+}(t)=0 \text { en } T^{-} ; \mu_{j}^{-}(t)=0 \text { y } \mu_{j}^{+}(t)=0 \text { en } T ; \mu_{j}^{-}(t)=0 \text { en } T^{+} ;
$$

de A2 se desprende que durante el intervalo en que la tecnología $j$ opera en el margen se tiene $P(t)=C_{j}$; integrando A2 en el intervalo $T^{+}$y teniendo en cuenta A1 se tiene:

$$
\left.I_{j}=\int \mu_{j}^{+} t\right) d t=\int\left(P(t)-c_{j}\right) d t
$$

Con el precio $P(t)$ durante el intervalo en que la tecnología $j$ trabaja a plena capacidad se recupera exactamente su costo de inversión.

En el caso de la tecnología $M$ de menor costo de inversión, que opera únicamente durante período(s) pico para suplir la demanda, no existe sino un instante en que opera a plena capacidad en el momento $\hat{t}$ en que ocurre el mayor pico, y no existen tecnologías con $C_{j}>C_{m}$. En este período además del costo marginal $C_{m}$ de la potencia se cobra un cargo igual al costo de inversión $I_{m}$ en capacidad con la tecnología $m$.

$$
L=\sum_{j=1}^{M} I_{j} Y_{j}+\sum_{j=1}^{M} \int_{t=0}^{T}\left\{\left[C_{j} Z_{j}(t)+P(t)\left[D(t)-\sum_{j=1}^{M} Z_{j}(t)\right]+\sum_{j=1}^{M}\left[-\mu_{j}^{-}(t) Z_{j}(t)+\mu_{j}^{+}(t)\left(Z_{j}(t)-Y_{j}\right)\right]\right\} d t\right.
$$

Las condiciones de optimalidad (Kuhn-Tucker) son:

$$
\begin{aligned}
& \partial L / \partial Y_{j}=I_{j}-\int_{0}^{T} \mu_{j}^{+}(t) d t=0 \\
& \frac{\partial L}{\partial Z_{j}(t)}=C_{j}-P(t)-\mu_{j}^{-}(t)+\mu_{j}^{+}(t)=0
\end{aligned}
$$

\section{REFERENCIAS}

Boiteaux, M. (1949). La Tarification des demandes en point: application de la theorie de la vente; cout marginal. Revue Generale de Electricité, (58) pp. 321-340

Borenstein, S., (2005). The Long Run Efficiency of Real Time Dynamic Electricity Pricing, Energy Journal (26) pp. 93-116 
Borenstein, S., \& Davis, L. W. (2013). Effective and Equitable Adoption of Opt-In Dynamic Electricity Pricing. Review of Industrial Organization, (42) pp. 127-160

Bye, R. T. (1926). The nature of Fundamental Elements of Costs. Quarterly Journal of Economics, (41) pp. 30-63

Crew, M, A., Fernando, C. S., Kleindorfer P. R. (1995). The Theory of Peak-Load Pricing: A Survey. Journal of Regulatory Economics, (8) , pp. 215-248

Durán H. (2001). Modelo de Precios de Período Pico con Tecnologías Diversas, presentado en el V Seminario sobre Regulación y Mercados de Energía, Universidad de los Andes, Universidad Nacional Medellín, UPME, COLCIENCIAS.

Joskow, P. L., (2012). Creating Smarter U. S. Electricity Grid. The Journal of Economic Perspective (26) pp. 1-21

Joskow, P. L., Wolfram, C. D. (2012). Dynamic Pricing of Electricity. The American Economic Review, (102) pp. 381-385

Lueberger, David G. (1989). “Programación Lineal y No Lineal". Adison-Wesley.

Steiner P. O. (1957). Peak Loads and Efficient Pricing. Quarterly Journal of Economics, (71) pp. 585-610 